\title{
Patientenverfügungen in der Psychiatrie - eine Chance
}

\author{
René Bridlera, Tobias Ballweg ${ }^{b}$
}

a Ärztlicher Direktor, Sanatorium Kilchberg AG; ${ }^{b}$ M.A. Phil., Dipl.-Psych., Leiter Ethikforum, Sanatorium Kilchberg AG

In der SÄZ ging Michael Kammer-Spohn kürzlich auf Wirkungen, Risiken und Nebenwirkungen psychiatrischer Patientenverfügungen ein und stellte ein Formular von Pro Mente Sana vor [1]. Der folgende Text streift die Literatur zur Bedeutung von Patientenverfügungen im psychiatrischen Kontext, unterstreicht die Relevanz ethischer Überlegungen und präsentiert eine eigene Vorlage, die sich in einigen zentralen Aspekten von derjenigen der Pro Mente Sana unterscheidet.

\section{«Patientenverfügungen in der Psychiatrie - Ärgernis oder Chance?»}

Was der Titel dieses SÄZ-Artikels von Michael Kammer-Spohn [1] als Frage aufwirft, ist in der Literatur weitgehend geklärt. Psychiatrische Patientenverfügungen greifen ein fundamentales Interessen der Patienten auf, ermöglichen Selbstbestimmtheit und fördern Empowerment. Empowerment meint die Erarbeitung eines individuell plausiblen Krankheitsverständnisses, Kenntnisse über Therapiewahlmöglichkeiten sowie das Zutrauen in die eigene Fähigkeit zur Steuerung des Gesundungsprozesses. Entgegen mancher Befürchtung sind Patienten durchaus in der Lage, verständliche und klinisch sinnvolle Anord-

Directives anticipées du patient en psychiatrie - un avantage

Le nouveau droit de la protection de l'adulte accorde une grande importance aux directives anticipées du patient. Alors que les directives somatiques portent généralement sur les situations de fin de vie, les directives psychiatriques règlent les phases cycliques d'incapacité de discernement pouvant résulter de troubles psychiques. En 2014, deux projets de directives psychiatriques anticipées ont vu le jour en Suisse. Le présent article met en lumière les différences entre ces deux projets et leurs conséquences respectives sur le quotidien clinique. Récemment, le BMS a publié un article de M. Kammer-Spohn sur les directives psychiatriques anticipées [1]. Son auteur y évoquait les avantages, les risques et les inconvénients de ces directives et présentait en détail un "formulaire» de Pro Mente Sana (PMS). Le présent article se penche quant à lui brièvement sur la littérature existante en matière de directives anticipées du patient dans le contexte de la psychiatrie, souligne l'importance des considérations éthiques et présente son propre projet, qui se distingue de celui de PMS sur certains aspects centraux. nungen zu treffen, die in aller Regel in Übereinstimmung mit Guidelines sowie der lokalen Behandlungspraxis stehen. Die Totalverweigerung jeglicher Medikation ist sehr selten. Barrieren gegen die Implementierung von Patientenverfügungen sind u.a. eine fehlende Unterstützung, ungenügende Kenntnisse der gesetzlichen Regelungen bei allen Beteiligten und negative Einstellungen der Professionellen. Kontrollierten Studien verdanken wir die Erkenntnis, dass eine geeignete Unterstützung bereits mit geringem zeitlichem Aufwand zur Errichtung von Patientenverfügungen und zu einer signifikanten Reduktion von späteren Zwangsmassnahmen führen kann (Übersicht mit Literatur bei [3, 4]).

\section{Die Patientenverfügung des Sanatoriums Kilchberg}

Die Erarbeitung der Vorlage des Sanatoriums Kilchberg folgte wie bei Pro Mente Sana (PMS) einem modernen Drehbuch. Sie entstand unter der Federführung des Leiters Ethikforum, einer Juristin und einem Patienten, dem Team gehörten zudem Ärzte und Pflegende an. Nach einer Vernehmlassung bei 20 Patienten und psychiatrischen Peers wurde die heute verfügbare Endversion erstellt [2]. Auf den ersten Blick scheinen die beiden Verfügungen ähnlich, unterscheiden sich aber in wesentlichen Aspekten, wie nachfolgend dargestellt.

\section{Maximierung der Autonomie in der Behandlungssituation}

In den letzten 50 Jahren hat sich das Prinzip der Autonomie als oberste ethische Maxime in der Medizin 
durchgesetzt. Kants Selbstzweckformel folgend, verbietet sich jede Instrumentalisierung eines Menschen [5]. Die Einlösung des Autonomieprinzips erfolgt im therapeutischen Alltag durch das Einholen der Einwilligung, den sog. «Informed consent». Hieraus ergibt sich das höchstpersönliche Recht des urteilsfähigen Patienten, jedes Heilverfahren abzulehnen, auch wenn es medizinisch indiziert ist. Diese Vetomöglichkeit bezeichnet man als kategorisches Abwehrrecht. Davon zu unterscheiden ist das nachrangige Wunsch- oder Erfüllungsrecht, dessen Verwirklichung sich anderen, ausserhalb der wünschenden Person liegenden Kriterien beugen muss, beispielsweise Gerechtigkeitsaspekten. Neben der Unterscheidung zwischen dem kategorischen Abwehr- und dem konditionalen Erfüllungsrecht sind weitere Differenzierungen des Autonomiebegriffs vorzunehmen. Willensäusserungen zu komplexen Sachverhalten, wie sie eine medizinische Behandlung darstellt, gelten dann als autonom, wenn die Person sie in einem Zustand der Freiwilligkeit, der angemessenen Informiertheit, der qualifizierten Urteilsfähigkeit und in Übereinstimmung mit ihren grundlegenden Wertvorstellungen (Authentizität) macht $[5,6]$.

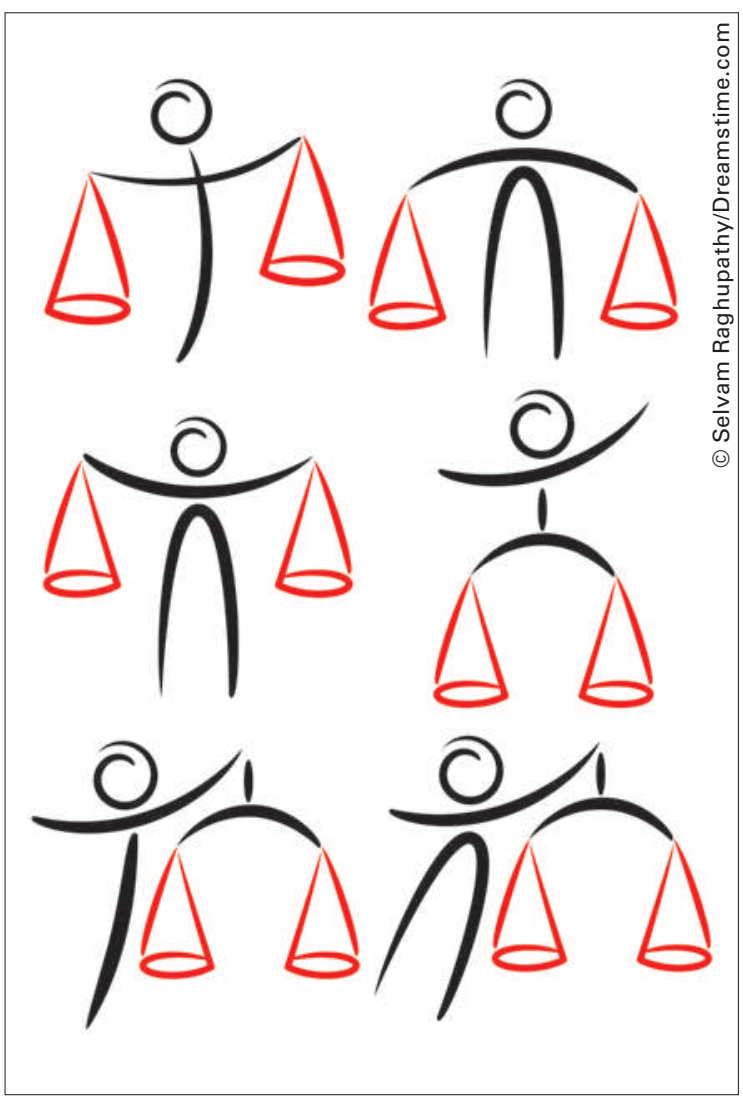

In allen Situationen die richtige Balance finden zwischen Patientenautonomie und Fürsorgepflicht des Arztes - eine Herausforderung besonders in der Psychiatrie.

\section{Verankerung des Autonomieprinzips in der Patientenverfügung}

Das primäre Ziel einer Patientenverfügung besteht darin, die Autonomie der urteilsunfähig gewordenen Person in der Behandlungssituation zu maximieren bzw. den Autonomieverlust zu minimieren. Eine idealtypische Vorlage sollte so strukturiert sein, dass die verfügende Person authentisch, frei von äusserem Druck, ihrem persönlichen Erfahrungs- und

Äusserer Druck kann auch durch die Struktur der Vorlage und die Art der Fragesteuerung entstehen.

Kenntnisstand entsprechend und in Übereinstimmung mit ihren Werten den eigenen Willen zum Ausdruck bringen kann. Äusserer Druck kann auch durch die Struktur der Vorlage und die Art der Fragesteuerung entstehen. Zur Vermeidung einer vorspurenden Denkeinengung (sog. "Priming») sollten allgemeine Werthaltungen vor konkreten Behandlungsaspekten, grundlegende Behandlungspräferenzen vor präzisen Therapiewünschen erfragt werden. Die Art der Fragestellungen in der Patientenverfügung sollte sowohl der verfügenden Person als auch den Anwendern im klinischen Alltag eine klare Unterscheidung ermöglichen zwischen expliziten Anweisungen (Abwehrrecht), expliziten Wünschen (Erfüllungsrecht, verbunden mit dem ausdrücklichen Hinweis auf eine bedingte Gültigkeit) und impliziten Wünschen und Anweisungen, die sich aus der Selbstbeschreibung ergeben.

\section{Authentizität und Informiertheit}

Der Beschreibung der eigenen Persönlichkeit mit ihren individuellen Werthaltungen und bisherigen Erfahrungen in Krisensituationen kommt eine besondere Bedeutung zu, weil sie zwei zentralen Aspekten des Autonomiebegriffs Rechnung trägt: Authentizität und Informiertheit (s. oben). Letztere beinhaltet nicht nur ein Wissen um die äusseren Bedingungen einer Behandlungssituation, sondern auch eine Kenntnis der eigenen Ressourcen und Einschränkungen, die sich in Krisensituationen bemerkbar machen. Aus deren Beschreibung ergeben sich für das Behandlungsteam wertvolle Hinweise, wie der Selbstbestimmtheit einer Person auch unter Gegebenheiten entsprochen werden kann, für die sie keine expliziten Anweisungen getroffen hat. Deshalb lässt die Patientenverfügung des Sanatoriums Kilchberg einer differenzierten Beschreibung der eigenen Per- 
sönlichkeit deutlich mehr Raum als die Vorlage der PMS. Schliesslich sollte eine Patientenverfügung dazu ermutigen, Präferenzen oder Ablehnungen zu begründen, weil dadurch die Motive der verfügenden Person verstehbar werden. Verständnis für die Motivlage erzeugt anerkanntermassen Wohlwollen und hilft bei der Suche nach Alternativlösungen.

\section{Die Bedeutung des Autonomieprinzips am Beispiel der Medikation}

Ein ethisch differenzierter Autonomiebegriff findet seinen Niederschlag auch bei expliziten Anweisungen und Wünschen, die zum Beispiel die Behandlung mit Psychopharmaka betreffen. Gerade hier ist die Gefahr von Autonomieeinbussen im Behandlungsfall besonders hoch. Die PMS-Vorlage fragt nach einer expliziten Zustimmung zu einzelne Substanzen, die im Falle der Urteilsunfähigkeit eingesetzt werden dürfen. Die Präparate werden in Tabellenform erfragt, einzutragen sind der Name, die gewünschte Verabreichungsform und - in Milligramm - die Morgen-, Mittags-, Abend- und Nachtdosis, die maximale Einzeldosis sowie die maximale Tagesdosis. Was die Willensautonomie hoch präzis zu erfassen scheint, entpuppt sich als problematisch. Zunächst fällt das

\section{Hätte die Person bei einer offen formulierten Frage die Behandlung ebenfalls auf $10 \mathrm{mg}$ eingeschränkt?}

Fehlen eines direkt platzierten Hinweises auf, dass nicht alle Rubriken ausgefüllt werden müssen. Weiter suggeriert der verlangte hohe Präzisierungsgrad in Verbindung mit dem unterstrichenen Wort $\mathrm{Zu}$ stimmung, dass es sich um eine besonders kritische Frage handelt, deren Beantwortung ein erhöhtes Mass an Vorsicht und Genauigkeit erfordert (im Gegensatz zu anderen Fragen). Dadurch werden sich auch diesbezüglich eher indifferente Personen zu Angaben gedrängt fühlen, was dem Gebot der Freiwilligkeit zuwiderläuft. Schliesslich täuscht der hohe Detaillierungsgrad die Fähigkeit einer punktgenauen Vorhersage zukünftiger Krankheitsepisoden und deren Behandlung (in Milligramm) vor. Dadurch wird nicht nur eine unrealistische Erwartung geweckt, sondern auch der Boden für fruchtlose Konflikte mit dem Behandlungsteam geschaffen. Unklar bleibt zudem, ob die verfügende Person mit der eingeforderten Zustimmung das Abwehr- oder das Erfüllungsrecht wahrnehmen möchte.

Zur Illustration stelle man sich folgende Situation vor: Eine Person trägt ohne weitere Begründung eine Medikation mit Olanzapin $10 \mathrm{mg}$ (O-O-O-1) in die vor- gesehenen Rubriken ein. In der akuten Behandlungssituation können sich folgende Fragen stellen: Wollte die Person damit die Zustimmung zu Olanzapin im Allgemeinen oder eine präzise Zustimmung nur zu $10 \mathrm{mg}$ zum Ausdruck bringen? Hätte die Person bei einer offen formulierten Frage die Behandlung ebenfalls auf $10 \mathrm{mg}$ eingeschränkt? War ihr das überhaupt wichtig? Warum hat sie $10 \mathrm{mg}$ und nicht beispielsweise nur $5 \mathrm{mg}$ oder sogar $15 \mathrm{mg}$ eingetragen? Sind $10 \mathrm{mg}$ möglicherweise die einzige Dosis, mit der sie bisher Erfahrung hat? Wollte die Person mit der Verfügung zum Ausdruck bringen, dass sie jede andere Dosis ablehnt, beispielweise 7,5 oder $15 \mathrm{mg}$ ? Wären Behandlungen mit 7,5 mg oder $15 \mathrm{mg}$ gleichermassen als Missachtung der Patientenverfügung zu verstehen?

\section{Dynamik und Unvorhersehbarkeit des Lebens}

Angesichts der hohen Dynamik jedes Lebens und der eingeschränkten Vorhersehbarkeit zukünftiger Krisen verzichtet die Kilchberg-Verfügung auf eine derartige Zuspitzung von Fragen. Die verfügende Person wird ersucht, gute und schlechte Erfahrungen mit einzelnen Medikamenten aufzulisten und belastende Nebenwirkungen in einer für sie stimmigen Reihenfolge anzugeben (implizite Anweisungen). In einer nicht weiter strukturierten Rubrik kann sie in freier Weise zusätzliche Bemerkungen zu den Medikamenten anbringen, beispielsweise ein bestimmtes Präparat ablehnen oder Dosierungswünsche äussern (explizite Anweisungen). Die dargestellte Fragesteuerung nutzt in optimaler Weise die Informiertheit des Patienten (beispielsweise betreffend Nebenwirkungen) als einen wichtigen Aspekt von Autonomie und erhöht so die Chance, in guter Übereinstimmung mit der Patientenverfügung eine sinnvolle und wirksame Behandlung durchführen zu können.

\section{Gesetz und psychiatrische Patienten- verfügung}

Wie bereits erwähnt, besteht eine wesentliche Bedeutung von Patientenverfügungen darin, einen Autonomieverlust im Behandlungsfall $\mathrm{zu}$ verhindern. Allerdings kann ein solcher Verlust auch durch eine Patientenverfügung mitverursacht werden - etwa dann, wenn eine Vorlage Entscheidungsmöglichkeiten suggeriert, die aufgrund der gesetzlichen Rahmenbedingungen nicht vorhanden sind. Dies lässt sich am Beispiel des Vertretungsrechts veranschaulichen. Gemäss Art. 370 ZGB kann im Rahmen einer Verfügung eine natürliche Person bestimmt werden, 
die im Falle der Urteilsunfähigkeit des Verfügenden mit den Ärzten die Therapiemöglichkeiten bespricht und abschliessend darüber entscheidet. Art. 378 ZGB zufolge steht diese vertretungsberechtigte Person an oberster Stelle einer mehrstufigen Kaskade mit der Befugnis, anstelle der urteilsunfähigen Person medizinische Massnahmen gutzuheissen oder abzulehnen (kategorisches Abwehrrecht). Mit Blick auf die Psychiatrie hat der Gesetzgeber das Vertretungsrecht jedoch eingeschränkt. Gemäss Art. 380 ZGB richtet sich die Behandlung eines urteilsunfähigen Patienten in einer psychiatrischen Klinik nicht nach Art.

Die Patientenverfügung des Sanatoriums Kilchberg lässt einer differenzierten Beschreibung der eigenen Persönlichkeit deutlich mehr Raum.

378 ZGB, sondern nach den Bestimmungen zur Fürsorgerischen Unterbringung (FU; Art. 426-39 ZGB), unter denen die Entscheidungen einer allfälligen Vertretungsperson rechtlich nicht bindend sind. Damit soll vor allem verhindert werden, dass Angehörige einen Vertretungsanspruch mit der Absicht geltend machen, eine urteilsunfähige Person in einer psychiatrischen Klinik dauerhaft unterzubringen. In einer Art gesetzgeberischer Kompensation stellt Art. 432 ZGB dem urteilsunfähigen FU-Patienten in der Psychiatrie fakultativ eine Vertrauensperson zur Seite, die ihn während der Unterbringung und bis zum Abschluss aller damit zusammenhängenden Verfahren unterstützen kann.

\section{Vertretungs- versus Vertrauensperson}

Die in Art. 370 ZGB erwähnte natürliche Person wird in der PMS-Verfügung als Vertretungsperson bezeichnet, die Kilchberger-Vorlage verwendet den Begriff der Vertrauensperson. Damit vollzieht sie die gesetzgeberisch gewollte Trennlinie zwischen Behandlungen somatischer Krankheiten einerseits und psychiatrischen Massnahmen unter FU-Bedingungen in der Psychiatrie andererseits (ohne sie deshalb gutzu- heissen). De iure ist die sogenannte Vertretungsperson - anders als die Benennung suggeriert - in der Psychiatrie unter FU-Bedingungen gerade nicht vertretungsberechtigt, sondern hat nicht mehr Rechte als eine Vertrauensperson. Dieser Umstand sollte durch eine verwirrliche Begriffswahl in der Patientenverfügung nicht verwischt, und es sollten keine Einflussmöglichkeiten dort suggeriert werden, wo sie nicht vorhanden sind.

\section{Fazit}

Psychiatrische Patientenverfügungen begünstigen Selbstbestimmtheit und Empowerment und können zukünftige Zwangsmassnahmen reduzieren. Ihr Ziel ist die Wahrung der persönlichen Autonomie in krankheitsbedingten Phasen der Urteilsunfähigkeit. Aufbau und Fragesteuerung der Patientenverfügung orientieren sich idealtypisch an einem differenzierten Autonomiebegriff, der die Persönlichkeit mit ihren individuellen Erfahrungen und Werthaltungen in den Mittelpunkt stellt, eine klare Unterscheidung zwischen Anweisungen (Abwehrrecht) und Wünschen (Erfüllungsrecht) vorsieht und keine Entscheidungen nahelegt, die entweder nicht realisierbar oder als ein Resultat allzu konkreter Formvorgaben gar nicht wirklich gewollt sind. Flexibel gestaltbare Patientenverfügungen können Konflikte in Akutsituationen verhindern und das Autonomieerleben der urteilsunfähigen Person fördern.

\section{Literatur}

1 Kammer-Spohn M. Patientenverfügungen in der Psychiatrie Ärgernis oder Chance? Schweiz Ärztezeitung. 2015;96(6):196-9.

2 www.sanatorium-kilchberg.ch/data/uploads/SK_Patientenverfuegung_0914_8759.pdf

3 Bridler R. Einen Schritt vor, zwei Schritte zurück. Das neue Erwachsenenschutzrecht und die Psychiatrie. Schweiz Ärztezeitung. 2013;94(12):486-9.

4 Bridler R, Gassmann J. Das neue Erwachsenenschutzrecht. Psych Pflege. 2012;18:183-77.

5 Maio G. Mittelpunkt Mensch: Ethik in der Medizin. 1. Korrigierter Nachdruck der 1. Aufl. Stuttgart: Schattauer; 2012

6 Beauchamp TL, Childress JF. Principles of Biomedical Ethics. 5th ed. New York, Oxford: Oxford University Press: 2001. 